\title{
Modelling and Simulation in Health Care Technology - from Development via Implementation to Assessment
}

\author{
Nikolas Popper ${ }^{1}$, Siegfried Wassertheurer ${ }^{2}$ \\ ${ }^{1}$ dwh Simulation Services Vienna, \\ ${ }^{2}$ Austrian Institute of Technology, Dept. Biomedical Systems, Vienna
}

SNE Simulation Notes Europe SNE 20(2), 2010, 5-14, doi: 10.11128/sne.20.on.09971

Modelling and Simulation (M\&S) has found its way into many areas, almost in our daily life, so also into health technology (HT). Health technology and medical technology encompass a wide range of health care products and services - from vaccination strategies to heart circulation monitoring. And they are used to diagnose, monitor or treat every disease or condition that affects humans. M\&S in HT is improving the quality of health care delivered and patient outcomes through earlier diagnosis, less invasive treatment and reductions of illness. This contribution investigates $M \& S$ in $H T$ at different levels: (i) $M \& S$ for developing a HT product, (ii) $M \& S$ implemented in a HT product, and (iii) $M \& S$ for assessment of a HT product. While the step from model-based development of HT products to model-based operation of HT products is evident, the last step to model-based assessment of HT products is relatively new application of M\&S - and necessary for the success of HT products. Interestingly, although the aims of M\&S at these three levels differ significantly, the M\&S techniques - from Markov models via ODEs/PDEs to Agent-based models stay the same.

\section{Introduction}

Experts in many application areas today use methods of modelling and simulation for developing new products and services. In many technical fields this is already well standardised and widespread in all areas from developing to implementation, and also for assessment of implemented solutions. In HT the usage of $M \& S$ has recently reached different stages of integration of the methodology in these areas. That means we can see different levels in the usage of modelling and simulation in development, implementation and assessment of HT. This situation gives us the possibility to focus on different aspects of M\&S, to analyse different possibilities and problems and furthermore we can demonstrate the various requirements for M\&S by reference to these areas of Health Technology Assessment.

Today development of new health technologies is assisted by many standardised models, like physical modelling, partial differential equations or compartment models. Also implementations can refer to many technical realisations over the last decades, which have led to standards or de-facto standards. Especially the usage of widely used platforms like MATLAB show a wide consensus in the community and lead to de facto standards, which - by the way - not always have to be the best possible solution.
Still methodical improvements on a market of highly competitive systems are important. New models are rated by how well the methodology can map and realise available data, the "state of the art" knowledge and established features - for example for mobile terminal devices. So - in the field of development and implementation of new Health Technologies - the discussions about detail-oriented improvements dominates the scientific discourse, sometimes losing the survey and the attendance for new, alternative approaches and solutions.

The situation in the area of the assessment of medical services is different. After the development of a new product or a new service these are assessed and their success is analysed and quantified. These assessments currently use $M \& S$ too, as e.g. the European Joint Actions (EUNetHTA) show; however the methodology is quite new and a lot of solved problems for technical areas remain open today. A main difference in the state of the art between M\&S in development and implementation and M\&S in assessment is that it is still under development which modelling method or which simulation implementation represents the best solution. So competition can here be found in a totally different area of model development. Missing standards lead to often not target-oriented discourse, which lacks a common language. 
In the scientific discourse we have two opposite situations. The different "roles" of M\&S in development, implementation on the one side and assessment on the other side are in the focus of considerations.

Are questions like the dominance of one modelling technique to be answered definitely at some point of development of $\mathrm{M} \& \mathrm{~S}$ in an application area like HT? Continuous research clarifies the demands of $M \& S$, so the choice of the M\&S Technique should also be clarified. Or are demands and techniques in a constant change, also changing the demands and possibilities for $\mathrm{M} \& \mathrm{~S}$ ? What are the minimum standards for an effective scientific discussion between different M\&S methodologies like PDEs and Cellular Automatons? How can demands for the use of M\&S be formalized to make the different techniques comparable.

Are there restrictions for the use of M\&S in HT? What is the primary objective of M\&S? Is it the construction of new things? Or is it the optimisation of implemented processes? Or is it the acquisition of a deeper understanding of a system? "Anything goes" one could say, but trust in a jack of all trades device has always been, and still is, subdued - and this is well justified. Models can and will be utilised for various - even opposing - aims. This constitutes an enormous potential, but on the contrary it involves the danger of "abuse".

\section{Models in Development of Health Technology Products}

One example for the need of new HTs are cardiovascular events. Recently here has been a rapid increase, however there has only been moderate corresponding success in the implementation of actual treatment strategies, with an unfortunate simultaneous dramatic rise in treatment costs. Actual data provided by the WHO or the American Heart Association accordingly show that about 50 percent of all deaths in OECD countries are brought about by some form of cardiovascular dysfunction. Hypertension can actually be considered as the most important pathology in cardiovascular disease.

Due to these facts medical sciences indicate an urgent need to find further relevant indicators besides high blood pressure. The current treatment guidelines of the European Cardiology Society (ESC) and the European Society for Hypertension (ESH) point out the need to analyse the cardiovascular system using pulse wave analysis (PWA) and the synthesis of hemody- namic parameters. However, the guidelines emphasize the lack of easy-to-use technologies. These, much needed technologies are, on the one hand noninvasive measuring devices and on the other hand models and tools for indirect measurement or modelbased determination of cardiovascular parameters.

Today Hypertension is seen as a relevant risk factor, but reasons for cardiovascular dysfunction are manifold and complex. Since risk factors cluster in hypertensive individuals, risk stratification should be employed and decisions on the management should not be based on blood pressure alone, but also on to the presence or absence of other risk factors, target organ damage, diabetes, and cardiovascular or renal damage, as well as on other aspects of the patient's personal, medical and social situation.

Due to the complexity and diverse coherence requirements of the cardiovascular system, a broad multidisciplinary knowledge is required to understand the biophysical as well as the medial causes. This has been reflected in the formation of interdisciplinary research groups to work on this problem over the last few years. So also virtual physiological modelling is seen as a powerful approach to improve the understanding of the complexity of the human physiology as a whole. Another problem caused by the intrinsic complexity mentioned above is that many results obtained in fundamental biomedical research (as with cardiovascular research) do not find their way into true clinical practice, mainly because the required bridges to show practical benefits are missing. This demands a new generation of multidisciplinary clinicians, educated by the means of integrative systems like provided by $M \& S$.

\section{Combining Models - Modelling arterial and left ventricular coupling for non-invasivemeasurements}

Nowadays different "high-end" measurement devices exist for the determination of hemodynamic parameters such as cardiac output and peripheral resistance. On the one hand there are non-invasive methods, such as the Doppler method or impedancecardiographs, whose drawbacks are the operator dependency and the costs. On the other hand there are invasive methods, such as the Gold Standard Thermodilution used also in combination with pulse contour analysis (PCA). The disadvantage of these methods is the invasiveness and the combined risks. Existing non-invasive pulse contour analysis methods are either dependent on personal data, such as age, height or weight or on an initial calibration. 
In Figure 1 the functional principles of pulse contour analysis methods using a personal calibration factor obtained by Indicator- or Thermodilution are illustrated.

The basic idea behind PCA is that the cardiac stroke volume is proportional to the area of the pressure curve during systole. To consider physiological boundary conditions like vessel diameter or arterial compliance as well as the cardiovascular status, an external calibration is needed and has to be repeated periodically. Currently the non-invasive assessment of functional hemodynamic parameters using simple and affordable devices for widespread application in internal medicine or by general practitioners is not possible. The goal is the development of an algorithm for a non-invasive, portable, easy-to-use and affordable device for measuring functional cardiovascular parameters based on a common oscillometric measurement using an occlusive blood pressure cuff.

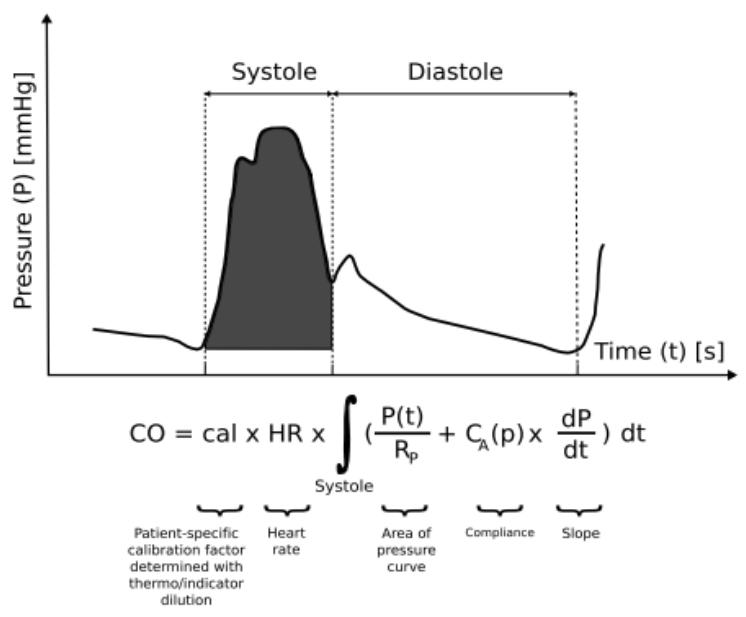

Figure 1. Example for cardiac output determination using pulse contour analysis.

An algorithm for a non-invasive, portable, easy-touse, and affordable device for measuring systemic cardiovascular parameters such as cardiac output and peripheral resistance was to be developed. The data acquisition was based on a common oscillometric measurement using an occlusive blood pressure cuff with no additional calibration necessary. For results and detailed description see [1]

This novel algorithm combines several simulation techniques like neural networks or differential equations. The determination of the hemodynamical parameters is based on the idea that the ejection work of the left ventricle is subject to an optimization principle.
As one advantage this kind of model needs no additional external calibration and opens therefore good perspectives for non-expert use in cardiovascular risk stratification and hypertension therapy optimization. Combination of different $M \& S$ techniques so lead to a simpler and more efficient tool.

The project showed evidence that the proposed method provides clinically relevant and reliable hemodynamics. The measurements showed good agreement between the algorithm and the reference methods. Therefore the application presents itself as a suitable alternative to standard cardiac output determination methods as well as a supplementary investigation method for general practitioners or specialists in internal medicine. Future work will incorporate additional considerations, regarding pulse wave velocity and pulse transit times, in order to refine the calculations on arterial compliance and subsequently reduce scattering and standard deviation with regard to the Thermodilution method.

\section{Models in Implementation - Pulse Wave Analysis}

As we could see a demand (development of an easier algorithm with less calibration effort) could be satisfied combining different M\&S techniques (see Fig. 2, Lattice-Boltzmann, Neural Networks, PDEs) to make non-invasive measurement more efficient. Another effort is to adapt measuring technology to new research results - new parameters have to be discussed - and to be modelled. The medical research regarding hypertension has fairly changed during the last two decades. Around the year 1990, the diastolic blood pressure was the most important value to look at, approximately 10 years later the focus was on the systolic blood pressure.

Today, we know that both systolic and diastolic blood pressures are prognostic ally important. But other vascular parameters seem to be of importance for evaluating the hypertensive patient with respect to his prognosis and potentially therapeutically options. With the beginning of the new millennium the topic of arterial stiffness of major vessels related to hypertension slowly arose in clinical practise. This issue was together with its indicators for the first time mentioned in the ESH-ESC (European Society of Hypertension- European Society of Cardiology) guidelines for hypertension treatment in the year 2003.

As parameters to measure arterial stiffness primary the methods of pulse wave analysis and pulse wave velocity have been suggested. 


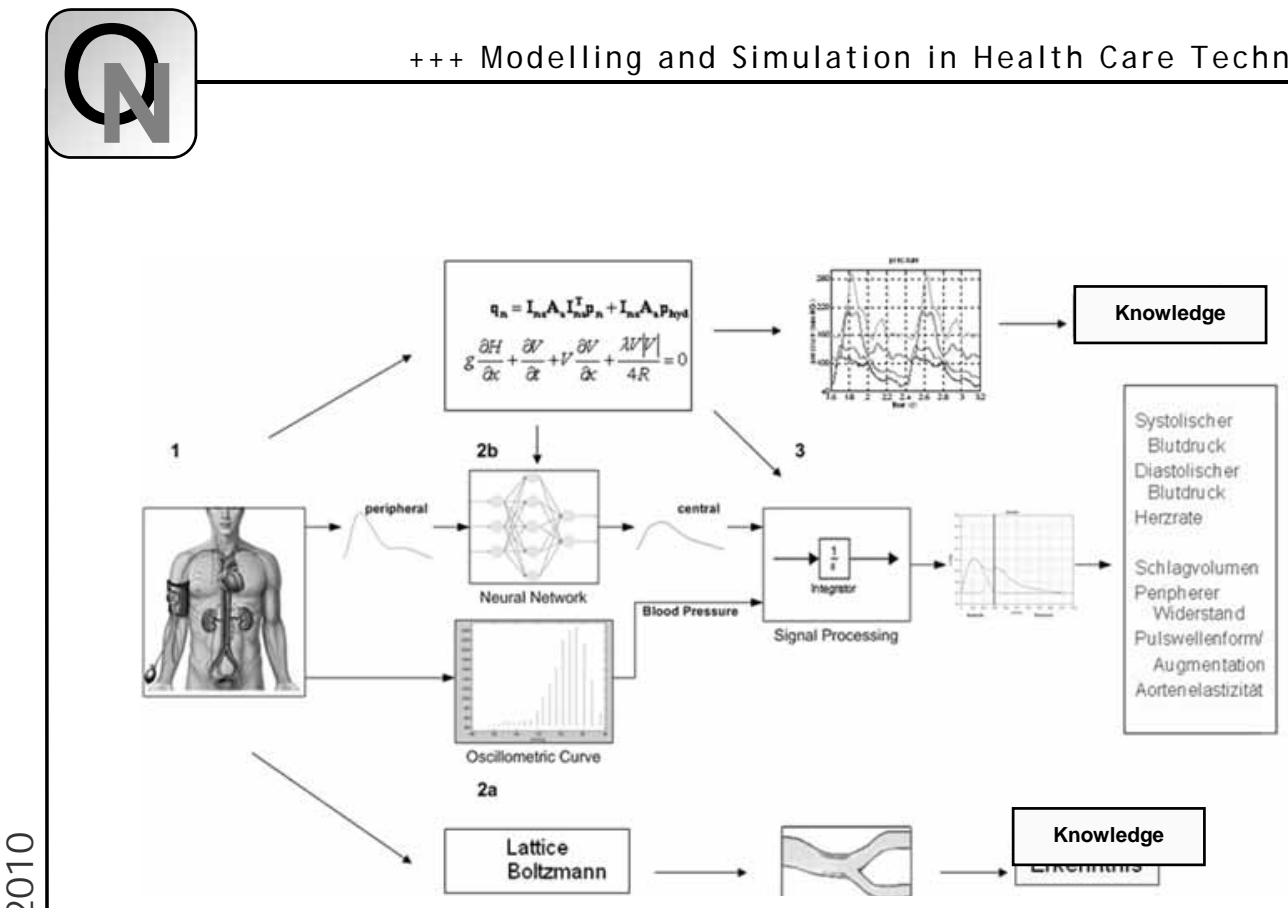

Figure 2 Structure of the strategy combining Techniques in developing process
Furthermore, it is reported that the AIx correlates with the left ventricular mass in normotensive men as well as in hypertensive ones. Moreover, the increase in cardiovascular risk can be estimated better from central than from brachial blood pressure measurements.

In the update of the ESHESC guidelines for hypertension treatment [2] in the year 2007, the consequences of arterial stiffness on cardiovascular mortality have a major role.
The pulse wave analysis evaluates shape and amplitude of the aortic pulse wave. The resulting parameters of relevance are the aortic systolic blood pressure (aSBP) and the so called augmentation index (AIx).

Owing to the differences in impedances between central and peripheral vessels and the moderate presence of wave reflection for healthy people, the systolic blood pressure at the aortic root is significantly lower than in the upper arm in such subjects. Diastolic and mean blood pressures do not differ significantly. 4 Different diameters and elasticities are responsible for the occurrence of these different wave impedances and the resulting differences in blood pressure. As a result of aging and pathological changes (for example, arteriosclerosis or subclinical organ damage) stiffening of vessels and therefore an increase of aSBP as well as an increased peripheral arterial resistance may occur. As a consequence of these changes, increased and premature pressure reflections emerge, which superimpose the generic pulse wave ejected by the heart earlier and more intensely. Their accumulation leads to an elevation of the aSBP and is called augmentation. The percent ratio of augmentation to the aortic pulse pressure is called AIx. The super- position may cause a pathological increase of the aSBP and subsequently an increase of the cardio- vascular risk.

It has been shown that both elevated aSBP and AIx are independent predictors of mortality in patients with end-stage renal disease and coronary heart disease.
These guidelines additionally claim to provide widely suitable measuring devices for the measurement of arterial wall stiffness and its influence on aortic blood pressure. The increase of the aSBP is ad hoc not noticeable in the A. brachialis and therefore per se not to be measured by common oscillometric methods. Therefore, the aim was the validation of the novel method determining AIx and aSBP based on an oscillometric method using a common cuff (ARCSolver, Austrian Institute of Technology, Vienna, Austria) against a validated tonometric system (SphygmoCor, AtCor Medical Pty Ltd, West Ryde, Australia)).

The ARCSolver method aims to be a novel method for the determination of the aSBP and AIx based on oscillometric blood pressure measurement with a common cuff. The method is the implementation of the development described above and uses the pulse waves assessed at A. brachialis. The recordings are carried out at diastolic pressure level for approximately 10s using a conventional blood pressure cuff for adults available in two sizes (24-34 and 32$42 \mathrm{~cm}$ ) and a high fidelity pressure sensor (MPX5050, Freescale Inc., Tempe, AZ, USA). The sensor is connected to a 12 bit A/D converter by means of an active analogue band bass filter $(00425 \mathrm{~Hz})$

After digitalization, the signal processing is performed using a three level algorithm. First, the single pressure waves are verified for their plausibility by testing the position of minima and the corresponding wavelengths. During the second stage, all single pressure waves are compared with each other to recognize artefacts. 
Thereafter, an aortic pulse wave is generated by the means of a generalized transfer function. The idea behind a transfer function is the modification of a certain frequency range within the acquired pulse signal to get the aortic pressure wave.

Comparison with published data showed similar results. The first positive zero crossing of the fourthorder time derivative of the generated aortic pulse wave represents the desired inflection point. Finally, the coherence of the measured parameters was verified. Therefore, the inflection point of each single pulse wave was compared with the mean inflection point. The determination of aSBP and AIx was carried out in the same way as in the reference SphygmoCor.

The aim of this comparison was the analysis for clinical suitability of the ARCSolver algorithms compared with SphygmoCor, which served as a reference device because of its wide distribution and acceptance. The parameters under investigation were the aSBP and the AIx. Both are surrogates for increased cardiovascular risk caused by increased and premature arterial wave reflections. The trials showed satisfactory accordance of the two methods. Thus, the ARCSolver algorithms are suitable for the use in oscillometric cardiovascular measuring devices. This is emphasized by the fact that the measurements were taken by a representative sample of healthy volunteers and patients during clinical routine (error bandwidths and measurement procedures if applicable).

The reasons for this good accordance may be based on the fact that for the transformation to determine the aortic blood pressure, the lower frequency bands of the pulse wave are dominant. This frequency bands are very robust and stable during measurement. The mentioned dominance may also blur the effects of high-frequency impedance changes between brachial and radial artery and its influence on central pressure. The results of the analysis of the AIx comparison show sufficient accuracy. The variation values determined in our studies are in the same range as those published for SphygmoCor. The ARCSolver is based on an user-independent recording method. Considering this, the measured values of the mean difference and the s.d. show consistency.

Using M\&S we were able to develop and implement new HT. Existing Technologies could be enhanced by combining different $\mathrm{M} \& \mathrm{~S}$ techniques.
This was on one hand important for improving the HT iteslf, but on the other hand research showed new demands (as other and more parameters are important for physicians, which partly can only be measured indirect) that can only be satisfied with new approaches.

\section{Modelling in Health Technology Assessment}

Austrian health care system spends about 20 to 25 million Euros every year. The social insurances, the federal states and the federal government pay a large part (about 70\%) of the costs. Because of new developments, which we described above - growing service portfolio and at the same time limited resources as well as an aging population lead to necessary decisions on supply of services. The assessment of new medical services that may or not may be paid by society - Health Technology Assessment - becomes more important every year.

Therefore the development of a comprehensible and problem-oriented assessment strategy for medical services both from a medical and economic viewpoint is necessary. Thus decision makers could better estimate costs and quality of medical services. In the long term a high level of medical service provision with optimal resource allocation will be ensured. The crucial factors for decision makers are flexibility, speed and replicability in the assessment - an ideal field of application for the application of models.

New studies show that between 4 and 8 percent of expenditures in the health care system are paid for administration, controlling, monitoring and decision support. For the small country of Austria alone this represents a market potential of (without expenditures for administration) 500 million Euros.

The application of models is most advanced in the area of development of new vaccination strategies. Vaccination is amongst the most effective prevention measures against infectious diseases. During the last years the use of vaccination has been discussed increasingly.

Each year many new vaccines are developed. Decision makers face the hard task of deciding on the reasonableness of each vaccination programme, primary with regard to cost-benefit efficiency. Comparison of competing vaccines in respect of costs and efficacy turns out to be especially difficult. 


\section{Demand of better decision foundations}

Recently health care decision makers realize that for example clinical studies cannot describe the whole process from spreading of an illness in a social network to analysis of courses and economical evaluations. Medical interventions based on wrong decision foundations waste resources. Considering the background that resources are limited and the political brisance of decisions concerning health care, decision makers need rational basis of decision-making.

Systematic and quantitative evaluation of patientrelevant and economic long-term consequences is required [3, 4]. Therefore the demand for more and better evaluations rises. With the help of optimal chosen and realized decision models using valid data bases, these evaluations and their results should be presented in comprehensible ways. New price models of vendors (so-called Risk Sharing Invoicing) enhance their need. Overall the application of models strengthens the position of decision makers towards vendors of medical services.

\section{State of the Art \& methodical restrictions}

Evaluation of vaccines is methodical particularly difficult. On the one hand modelling the spread of a disease needs to consider dynamic epidemiologic effects like herd-immunity, on the other hand social structures and costs must be considered appropriately. Evaluation of vaccination programs needs interdisciplinary assessment on the representation of the illness, whether the acute infectious aspect of the disease and therefore the epidemiological spread of the pathogen, which requires so-called dynamic models, or if the chronic aspect of the illness is in the foreground. Altogether it is necessary to choose suitable models and if it is required even connect several different models.

Apart from evaluations of databases and studies decision makers want progressively more systematic reviews of medical services and technologies with the help of Health Technology Assessment (HTA). HTA uses well-established and long proved methods to systematically evaluate medical services and assess the consequences of health care interventions. Independence of HTA-experts of medical vendors is essential. Recently developed HTA-principles [5] or guidelines of the German IQWiG to include Health Technology Assessment into the decision process emphasize this aspect.

\section{Necessity of Modelling \& Simulation}

Combination of classical meta-analysis of clinical studies, statistical methods and HTA with a flexible choice of models and calculated scenarios leads to a modern evaluation tool for medical services.

On the European level the three-year Joint Action Project EUNetHTA started on the 1.1.2010 shows the demand. EUNetHTA standardizes methodical aspects of the evaluation of medical services and for the first time tries to include modelling and simulation sustainable into the evaluation process.

Concretely the realization of appropriate models poses some questions which could not be solved methodically in a sustained manner. On the one hand it is not clear which modelling approach is optimal for which area because the whole previously described process had to be reproduced. Even when only examining the example of the evaluation of vaccination strategies the following aspects have to be realized in the model:

- Social structures and interactions between affected people

- Modelling transmission of an infectious disease including Serotypes, Herd Immunity et al.

- Integration of statistics of given or comparable countries about occurring illnesses and complications caused by the pathogen.

- Economic evaluation of these effects including standardized values from HTA

Apparently choosing and combining the best possible modelling approaches and realizations of different implementation possibilities lead to various problems.

In the area of Health Technology Assessment only classical respectively non-dynamic modelling approaches are used nowadays. These methods include Decision Trees, Markovian Models and System Dynamics. All of them are thoroughly discussed in literature (for example System Dynamic Models [6]). These approaches often emphasize the use of data but comparison of different models rarely happens.

Regarding modelling there are far more advanced modelling approaches which often lack the connection to the complex data structures. Even simple prediction and evaluation models put interdisciplinary working research groups in front of serious problems [7]. 
For example just to mention the various standardizes values like LYG (Live Years Gained) have to be expressed as a model result. The example of the development of a tool for evaluation of vaccination programs against pneumococci shows proposals for solutions and problems of the use of modelling and simulation in the evaluation of medical services.

\section{Assessment of a Vaccination Strategy}

In a cooperation of the independent institutions HVB (Main Association of Austrian Social Insurances), VUT (Vienna University of Technology) and dwh simulation services the socio-economic influence of including Prevenar (PCV7) pneumococcus children vaccine into the Austrian children vaccination program was modelled and analysed [8].

Different Model Types were implemented - on one hand to match, compare and validate with given study results and on the other hand to introduce a new way of modelling of future scenarios: The models were compared to each other and to the studies and can integrate various different data types and sources to compute potential future scenarios.

This "modular" model realises the opportunity to compute different scenarios for different aspects of the vaccination with various assumptions to compare results and stability of results for each module separately. Effects of changes - in population structure or size, epidemiological assumptions like serotype changes or herd immunity, new data for diseases or costs can be implemented to compute the dynamic future scenarios and to compute the future economic data for different treatments.

White Box Modelling guarantees not only the computed results but also the possibility of improving the knowledge about the model system and to re-evaluate the assumptions after every scenario. White Box Modelling offers the possibility to get additional information which data and in which way this data is needed for future studies.

\section{Analysis of the socio-economic influence of the introduction of the vaccination}

Using the general available vaccination distribution assumptions in Austria and the saved data of regulatory authorities, information concerning pop data and costs of hospitalization for pneumococcal disease one can yield detailed estimations regarding the total costs: in the vaccination setup time the annual costs increase to approximately $14.2 \mathrm{Mio}$ € and then de- crease due to the increasing number of avoidable cases per year to 12 Mio. €. This amount remains constant in the long run.

In spite of this deterrent high amount it is important to point out the accessible positive effects of the reduced burden of the disease: While in the first year after the introduction of the vaccination only about 15 critical pneumococcal illnesses can be avoided, after the first 10 years a positive effect of approximately 125 avoided meningitis-, sepsis-, and pneumococcal pneumonia cases can be expected. Especially, the possibility to prevent about 10 cases of meningitis a year has to be focused on.

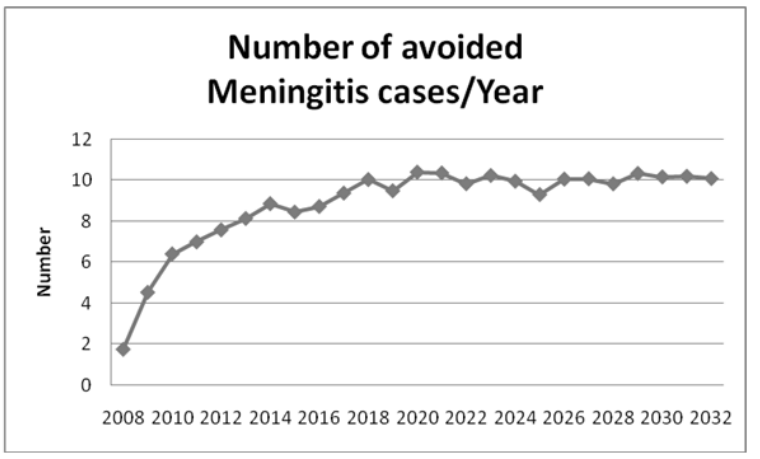

Figure 3 Number of avoided Meningitis Cases p.Y.

Another measure for the efficacy of vaccination programs is given by the abstract value CLYG, which are the costs per life-year gained. For the actually evaluated procedure the CLYG results in about 205.000€/LYG in the steady state.

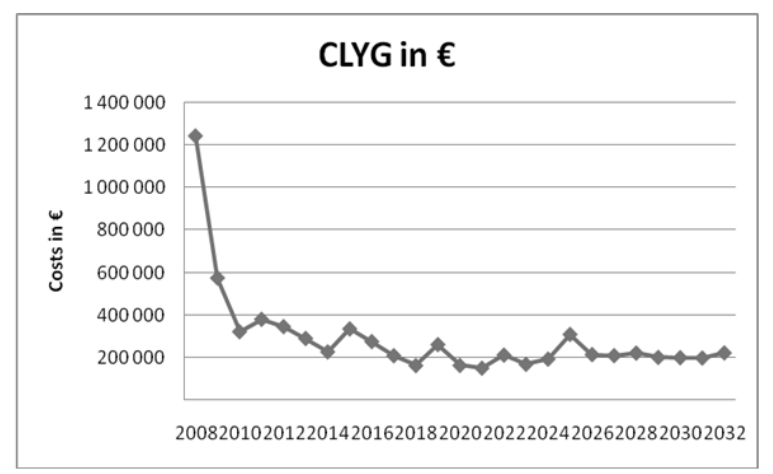

Figure 4 Costs per Life Year Gained in Euro

\section{Combining the Methods for international comparable results}

The results of this project are based on literature research, application of classical methods and on newly developed complex mathematical models, 
which are calculated for the first time for the spread of pneumococcal bacteria and show the influence of population structures, social behaviour of individual persons and epidemiological detailed knowledge in a dynamical model. For epidemiological purposes the innovations of this simulation model are the consideration of the dynamical structures of the population for the following years and, especially, the research concerning the effects of herd-immunity, which are positive effects for the unvaccinated population based on the decreased number of carriers due to the vaccination, and the serotype-shift, which refers to the influence of the chancing selection-impact on competing pneumococcal strains due to the vaccination.

To yield international comparable results and to classify possibilities and problems the first step was the implementation of the classical approach. Afterwards, assimilable dynamical methods were realized and modularly extended by further influencing factors. Other than in classical static methods the parameters for herd-immunity are not given but are modelled implicitly by the model structure and the possibility to comprise real social behaviour and, thus, agedependent contact between individuals including the possible risk of infection. Thereby, it is ensured not to depend on abstract estimators, which are based on not evaluable assumptions instead of studies with persons due to the long action time.

\section{Features}

Because of the detailed highly parametrisable and objective model structure vaccines can be compared (which is most times not possible in clinical studies) in the model and different vaccination strategies and scenarios can be tested. Furthermore, the mapping of individual abstract persons enables an extendable implementation of social behaviour as well as the analysis of influences concerning demographical changes of the next decades. Immigration and emigration are already accounted for in the base model. Scenarios for vaccinations strategies can be computed.

Another important factor for the supplier and the epidemiological analysis which can only be analysed using dynamical models is the vaccination setup phase. Other than in any vaccination for the populace for introduction of a vaccination in the children immunisation schedule the complete herd-immunityeffect and the maximal immunisation protections appears with the aging of the children- in this case it lasts about ten years.
The present simulation model provides an objective independent support for decision makers due to its open comprehensible structure (White Box Modelling) and the broad range of implemented dynamical non-linear effects like Serotype Changes and Herd immunity. Because of the modular build up demography, epidemiology and economical key decision values can be analysed separately and additional understanding of the problem can be supported.

\section{Summary of the benefits of this way of problem modelling and evaluation}

Long term population dynamics: Long term effects like carrier behavior and strategies can be focused on in detail with a high resolution timescale.

Start-up phase simulation: In contrast with classical Markovian or Decision Tree based models, dynamic modelling represents also the start up phase of the vaccination program. This is of main interest for decision makers, because in this timespan full vaccination cost occur, but on the other hand not the ideal benefits can be reached.

Herd immunity effects are model generated and time dependent: Herd immunity is generated during simulation time and is based on the social interaction of the modelled agents. Using a sophisticated model for carrier and an extra part for illnesses guarantees the capability.

Selection pressure is model generated: As mentioned for herd immunity, this dynamic nonlinear effect is also generated during simulation time and uses the advanced simulation technique and its flexibility.

Different scenarios: Due to well defined parameter ports, different scenarios can be calculated easily without changing model structures. Furthermore simulation runs for other countries can be implemented easily.

Long term prognosis: Referring to population dynamics and other external long term effects parameter gateways are implemented in the model and thereby, flexible analysis timespans can be calculated.

Comparison of different vaccines: Because of modelling the carrier rates in detail, the influence of different vaccines (serotype coverage, vaccine efficiency, ...) can be evaluated. Beyond also mixing of different vaccination strategies and the global influence can be represented. 
Adaptivity for different countries: Not only the poor parameter changes for foreign countries can be simulated, but also different assumption regarding social system structures.

White Box Modelling: One of the main benefits is that besides a complex high quality model used, the parametrization and used structure are dealing only with intuitive data and thereby the model can be used in interdisciplinary working groups.

In addition to the mentioned benefits of the implemented approach mainly fundamental open questions come up that need to be discussed in the following years.

For the evaluation of medical services, standardization of the used models is absolutely necessary. To that end consensus regarding the process of selection and the valid combination of appropriate modelling techniques have to be found in the scientific community. The European-wide acceptance of further development of models in the field of HTA in context of the EUnetHTA highly depends on this issue. Therefore a process has to commence that aims the comparability of different model approaches eventually.

The need for standardization becomes clear through consideration of the impact of such an assessment. Considering the economic impact the model described above leads to decision on cost in the range of 15 million Euros in the case of the use by decisionmakers [9].

But besides the financial aspects the decision on the use of medical services further plays an important role in the aspect of the future question of coexistence in our society: "Who has got the right to receive which performances?" One's health is probably one of the most central aspects of personal life and the gap between newly developed facilities and their "no longer be financed"-aspect has the potential to lead to enormous social conflicts.

The model approaches in the field of health economics, HTA and Evidence Based Medicine are currently mostly data-driven approaches. In contrast methoddriven approaches from the field of mathematics, computer science and physics cause a clash with the methods that are considered to be standard. The above-described method to compare models is only a very first small step of the necessary convergence of those different worlds.

\section{Conclusion}

HT touches some of the most important human needs: To keep a good health as long as possible, to anticipate future health problems or to cure illness. $M \& S$ helps HT to improve its "portfolio" in many ways. M\&S extends the possibilities for example in computing values, which are not or only very complicate to measure. Or $M \& S$ helps comparing vaccines which were not tested against each other.

It is clear that still modelling systems and simulating scenarios is always only the second best solution. It is always some kind of placeholder, until the real tests can be made or the real data is available. But until that time it is simply the „best" solution, and this shows that it is important to face the problems, which are raised by new and advanced modelling technologies.

New challenges for M\&S can only be solved by using new approaches. In some areas we have reached the boundaries of the state of the art methods. The tasks described above show that new structural information or new data lead to new structures of the model and so there may be need for new modelling techniques to cover this demand. In progressing technologies like HT you never can give a final conclusion which modelling technique is best.

To compare "state of the art" M\&S techniques and other approaches standards have to be developed. These strategies are followed by various scientific clusters and international efforts are made. Still it is to swim against the stream, as provider of modelling concepts and even more software developers have no interest to change a "winning team" - even if the game has changed.

New M\&S strategies - and most notably the extension of the application areas in HT - induce an even higher responsibility for validation of the implemented models and simulations. Comparison of different models might be a promising strategy to cope with this problem.

Finally, let us point to a more philosophical aspect and to the dependency and the difference using $M \& S$ for the areas mentioned in the title. Only development of new technologies - enabled also through M\&S leads to the need of Assessment. For which - of course $-M \& S$ is very useful. So solution of problems induce new problems that are even more complex than the previously solved one. But it even gets worse. 
Using M\&S for development we expect the solution of a problem, otherwise the model is discarded. Not so using $\mathrm{M} \& \mathrm{~S}$ for assessment. Here we must accept that the "non-decidability" of topics has to be accepted under certain circumstances. However neither the demand of the decision is waived nor is it compulsory to mistrust the quality of the model.

The traditional "Tertium non Datur" (Law of the Excluded Middle) does simply not apply. The first who introduced this well-known point against the universality of the law of excluded middle was already Aristotle [10]. He mentioned that the principle of the excluded middle does not apply to statements about the future (like the phrase "Tomorrow will be a sea battle") because the progress of the future is still open. And even if we try hard predicting the future using $M \& S$, sometimes even modern live is like a sea battle. Why?

Using M\&S we try to predict the future, but we do not succeed always. But decision makers have to decide - if they are not able to do this - even based on the best models and future forecasts - the discontent of the people might increase. In former times this led to the expulsion or even loss of life of decision makers, today fortunately probably only to the loss of personal power and influence.

Only one point can comfort us: Over time, the additional benefit of system knowledge and data, exclusion of errors and new evidence might ultimately lead to the decidability of the question [11]

\section{References}

[1] Wassertheurer S., Mayer C., Breitenecker F. : Modeling arterial and left ventricular coupling for non -invasive measurements. Simpat 2008; 16 : 988-997.

[2] Mancia G., de Backer G., Dominiczak A., Cifkova R., Fagard R., Germano G. et al. : Guidelines for the management of arterial hypertension. Eur Heart J 2007; 28: 1462-1536.

[3] Siebert U.: When should decision-analytic modeling be used in the economic evaluation of health care? [Editorial]. European Journal of Health Economics, 4, 143-150.
[4] Drummond M. F., Schwartz J. S., Jonsson, B., Luce B. R., Neumann P. J., Siebert U., Sullivan, S. D.: Key principles for the improved conduct of health technology assessments for resource allocation decisions. Int J Technol Assess Health Care, 24, 244-58.

[5] Franke R., Hart D.: HTA in den Entscheidungsprozessen des IQWiG und G-BA. Bundesgesundheitsblatt - Gesundheitsforschung - Gesundheitsschutz, Kategorie: Leitthema: HTA: Was verbirgt sich dahinter?. Volume 49, Number 3/März 2006

[6] Brailsford S.: System Dynamics: What's in it for healthcare simulation modelers, Winter Simulation Conference 2008.

[7] Ponholzer A., Popper N.,Breitenecker F., Schmid H. P., Albrecht W., Loidl W., Madersbacher S. , Schramek P., Semjonow A., Rauchenwald, M. : Proposal for a Standardized PSA Doubling-time Calculation, Anticancer Research, International Journal of Cancer Research and Treatment, Volume 30, Issue No.5

[8] Endel G., Schiller-Frühwirth I., Popper N., Zauner G., Breitenecker F.: Comparison of Different Static and Dynamic Simulation Techniques for the Influence of Children Pneumococcal Vaccination. Podiumsvortrag, ISPOR 12th ANNUAL EUROPEAN CONGRESS (Abstract published in Value in Health, 12(7), A233).

[9] Zauner G., Popper N., Schiller-Frühwirth I., Endel, G., Breitenecker, F.: Pneumokokkenimpfung für Kinder, Simulations-ergebnisse. EBM-Bericht, Hauptverband der Österreichischen Sozialversicherungsträger, 2009.

[10]Aristoteles: Kategorien, Hermeneutik oder vom sprachlichen Ausdruck, (2nd Book Organon) üs. von Hans Günter Zekl, Meiner, Hamburg 1998

[11]Bacon F.: Neues Organon (Novum Organum) Reihe: Philosophische Bibliothek 400/a Deutsch, Lat.. Meiner Felix Verlag GmbH,Januar 1990

\section{Corresponding author: Niki Popper}

dwh Simulation Services, Neustiftgasse 57-59

1070 Vienna, Austria

niki.popper@dwh.at

Received June 10, 2010 (Draft Inv. Lecture EUROSIM 2010)

Revised: August 15, 2010

Accepted: August 15, 2010 